\title{
Factores sociodemográficos asociados con los síntomas depresivos en una muestra de mujeres recluidas en dos prisiones de México
}

\author{
Frank Pulido-Criollo, ${ }^{1}$ Juan Francisco Rodríguez-Landa ${ }^{1}$ \\ y Martha P. Colorado-Martínez ${ }^{2}$
}

Forma de citar Pulido-Criollo F, Rodríguez-Landa JF, Colorado-Martínez MP. Factores sociodemográficos asociados con los síntomas depresivos en una muestra de mujeres recluidas en dos prisiones de México. Rev Panam Salud Publica. 2009;26(3):209-15.

RESUMEN Objetivos. Determinar la frecuencia de los sintomas depresivos en una muestra de mujeres recluidas en dos prisiones mexicanas e identificar los factores sociodemográficos asociados con estos sintomas.

Métodos. Se realizó un estudio exploratorio y descriptivo en mujeres de 18 a 50 años de dos prisiones del estado de Veracruz, México: los penales Pacho Viejo (PV) y Fortaleza San Carlos (FSC). Se aplicó la escala de depresión de Hamilton y se relacionó su puntaje con las características sociodemográficas de las participantes (edad, estado marital, número de hijos, actividad laboral dentro del penal y número y frecuencia de visitas que recibía) y su situación jurídica, tipo de delito cometido y tiempo de sentencia. Se utilizaron las pruebas de la $\mathrm{t}$ de Student, de la $\chi^{2} y$ la post hoc de Student-Newman-Keuls, según las características de las variables.

Resultados. Todas las mujeres estudiadas en ambos penales presentaron síntomas depresivos. Las mujeres de PV tuvieron un puntaje menor de sintomas depresivos que las de FSC $(20,8 \pm 0,8$ frente a 25,2 $\pm 0,9 ; \mathrm{P}<0,001)$. Se encontró un mayor puntaje de depresión en las mujeres con hijos que en las que no tenian hijos $(25,3 \pm 0,9$ frente a 21,8 $\pm 1,3 ; \mathrm{P}<0,05)$; el grupo de mujeres que recibía visitas semanalmente presentó un puntaje $(15,0 \pm 1,3)$ significativamente menor que los grupos restantes $(F[4,46]=30,7 ; \mathrm{P}<0,0001)$. No se encontraron diferencias significativas asociadas con el estatus marital, la situación laboral, la edad, el tipo de delito y el tiempo de sentencia.

Conclusión. Se encontró una elevada frecuencia de sintomas depresivos en la muestra estudiada; la mayor intensidad se relacionó con tener hijos y recibir visitas con poca frecuencia. Los programas de readaptación social deben fomentar el contacto permanente de estas mujeres con sus hijos, familiares y amigos.

Palabras clave Salud de la mujer; prisiones; trastorno depresivo; apoyo social; México.

Instituto de Neuroetología, Universidad Veracruzana, Xalapa, Veracruz, México. La correspondencia se debe dirigir a Juan F. Rodríguez-Landa, Apartado postal 391, Xalapa 91001, Veracruz, México. Correo electrónico: juarodriguez@uv.mx

2 Programa Estatal de la Mujer, Gobierno del Estado, Xalapa, Veracruz, México.
En el año 2000 había aproximadamente 9 millones de prisioneros en el mundo (1) $\mathrm{y}$, aunque hay más hombres que mujeres en prisión, en los últimos años ha aumentado el número de mujeres que ingresan a las cárceles (2-5). En la población peniten- ciaria prevalece un alto índice de trastornos mentales, como psicosis, ansiedad y depresión mayor (6-8), más frecuentes en mujeres que en hombres $(5,9)$.

Al parecer, el estrés psicosocial generado durante el encarcelamiento afecta 
más a las mujeres que a los hombres (4, $10)$, lo que contribuye a la alta incidencia de trastornos mentales en las mujeres encarceladas (4). Además de perder su libertad y de estar lejos de su familia, con frecuencia las mujeres encarceladas resultan abandonadas por sus familiares y amigos $(11,12)$. Según varios autores, la modificación de sus roles como mujer, madre y esposa, el confinamiento en un ambiente carcelario de intensa vigilancia, la inequidad en el proceso de readaptación y la limitada interacción social entre las mujeres recluidas en prisión las mantienen en un aislamiento social que provoca y agudiza algunos trastornos mentales, como la ansiedad y la depresión $(4,5,10,13,14)$.

Algunos estudios coinciden en que algunos factores sociales y características sociodemográficas, como el apoyo social, el sexo, la edad y el estatus marital y parental, entre otros, se relacionan con los síntomas depresivos, tanto en la población general como en la que cumple prisión (7, 15-17). De hecho, las mujeres encarceladas de 18 a 27 años, solteras, con hijos y con insuficiente apoyo social son las que con mayor frecuencia presentan trastornos depresivos (10, 16-18).

Identificar la posible relación entre los factores sociales y la presencia de síntomas depresivos en las mujeres que guardan prisión podría contribuir a desarrollar estrategias dirigidas a reducir el impacto del encarcelamiento sobre su salud mental y a esclarecer el motivo por el cual las mujeres suelen tener peor desempeño en las escalas de síntomas depresivos que los hombres, tanto en la población general como en la que se encuentra en prisión. Si se toma en cuenta que los factores sociales en general $-y$ los que provocan mayor estrés en particular- pueden ser causa de depresión (19), intervenciones bien orientadas podrían ayudar a reducir los efectos del estrés sobre el estado emocional de las personas y mejorar su calidad vida.

Si bien diversos estudios destacan la alta incidencia de los trastornos mentales en la población que guarda prisión y señalan que las mujeres son las más afectadas $(4,5,9,14,20)$, en México no se dispone de suficiente información al respecto y se desconoce la incidencia de trastornos mentales en las mujeres internadas en cárceles y la influencia en ello de los factores sociodemográficos. El objetivo del presente estudio fue determinar la frecuencia de los síntomas depre- sivos en una muestra de mujeres recluidas de dos prisiones mexicanas e identificar los factores sociodemográficos asociados con estos síntomas.

\section{MATERIALES Y MÉTODOS}

Se realizó un estudio exploratorio y descriptivo en dos prisiones del estado de Veracruz, México: los penales Pacho Viejo (PV) y Fortaleza San Carlos (FSC). El penal PV, localizado aproximadamente a $10 \mathrm{~km}$ al sur de la capital del estado, se construyó especialmente para funcionar como reclusorio, por lo que cuenta con los espacios necesarios para la readaptación social de los internos. El penal FSC, ubicado aproximadamente a $50 \mathrm{~km}$ al oeste de la capital del estado, se acondicionó en un antiguo fuerte que data del siglo XVIII, por lo que carece de los espacios necesarios para la readaptación social. Las poblaciones internas de ambos penales están compuestas por hombres y mujeres que comparten áreas de convivencia, pero tienen dormitorios separados. En ambos penales se les permite a las madres tener a sus hijos en la prisión hasta la edad de seis años.

La muestra (no probabilística) quedó compuesta por las que aceptaron participar en el estudio. Se excluyeron las mujeres mayores de 50 años, las que tuvieran alguna incapacidad física o enfermedad física crónica incapacitante, las embarazadas y las lactantes. Se eliminaron de la investigación los datos de las mujeres que durante el estudio decidieron retirarse, resultaron embarazadas, adquirieron alguna enfermedad física incapacitante, no ofrecieron toda la información requerida para el estudio o quedaron en libertad.

Después de informar a las autoridades de los penales sobre el proyecto y explicarles sus objetivos y alcance, se les informó del mismo a todas las mujeres en prisión y se solicitaron voluntarias. Previo a la firma del consentimiento, a las voluntarias se les informó acerca de los objetivos de la investigación y que podían retirarse del estudio cuando así conviniera a sus intereses, sin que esto tuviera alguna repercusión en su situación jurídica dentro del penal. Además, se les garantizó que sus datos personales se tratarían con estricta confidencialidad y anonimato y que los resultados que se obtuvieran del estudio serían utilizados solamente para los fines de la investigación.

De manera aleatoria se programaron sesiones individuales en las que un ex- perto en salud mental aplicó la escala de depresión de Hamilton y se recabaron datos sociodemográficos de cada participante: edad (en cuatro grupos: 18-20, 21-30, 31-40 y 41-50 años), estado marital (con pareja o sin pareja; con pareja dentro o fuera del penal), número de hijos (dentro o fuera del penal), actividad laboral dentro del penal, número de visitas que recibía y frecuencia de las visitas (semanales, quincenales, mensuales, menos de una visita al mes y no recibía visitas). Estas entrevistas duraron 20 minutos como promedio. La información relacionada con su encarcelamiento (situación jurídica, tipo de delito y tiempo de sentencia) se obtuvieron directamente de las autoridades del penal, a fin de no influir en el estado emocional de las entrevistadas. El tiempo de sentencia se analizó según tres grupos: 1-10, 11-20 y 21-30 años.

La escala de depresión de Hamilton, en su versión reducida de 17 preguntas, originalmente escrita en inglés y validada y adaptada al español en 1986 (21), se utiliza ampliamente para evaluar la intensidad o gravedad de los síntomas depresivos (22). Nueve preguntas se calificaron mediante una escala de 0 a 4 puntos, mientras que ocho se calificaron con una escala de 0-2; la suma máxima era de 52 puntos y se consideró que había síntomas de depresión a partir de 10 puntos.

Estudios de validez y confiabilidad de la escala de depresión de Hamilton, tanto de la versión original como de su versión en español, mostraron una alta correlación (entre 0,84 y 0,90 ) con las evaluaciones clínicas en pacientes psiquiátricos, independientemente de que presentaran depresión o no (23). Asimismo, se ha encontrado una alta correlación de hasta 0,77 entre la escala de depresión de Hamilton y las escalas de depresión de Beck y de Carroll, con una elevada consistencia interna (coeficiente alfa de Cronbach de hasta 0,85) (24). La versión en español de la escala de depresión de Hamilton presentó una alta correlación con la escala de depresión de Carroll ( $r=0,77 ; P=0,001)$ en una muestra de pacientes mexicanos que cumplían los criterios diagnósticos de depresión según la tercera edición del Manual Diagnóstico y Estadístico de los Trastornos Mentales (DSM-III); la confiabilidad temporal analizada mediante el procedimiento conocido como prueba-reprueba fue de $0,72(P=0,01)$ y el valor del coeficiente alfa de Cronbach para el total de 
las evaluaciones fue de 0,85 (24). Esta escala también se ha aplicado en la población mexicana (25) y en mujeres en prisión (26), con una aceptable correlación con los resultados de las entrevistas clínicas estructuradas basadas en la versión reducida del DSM-III.

\section{Análisis de los datos}

Para tomar en cuenta las diferentes características geográficas y arquitectónicas de cada uno de los penales, se realizó un análisis comparativo preliminar entre las características sociodemográficas y los puntajes de los síntomas depresivos de las mujeres de cada penal. Se utilizó la prueba de la $t$ de Student para comparar las variables cuantitativas que siguieron una distribución normal (edad, número de hijos, número de visitas, años de sentencia y puntajes en la escala de depresión de Hamilton) y la prueba de la $\chi^{2}$ para comparar los porcentajes de las mujeres agrupadas según sus características sociodemográficas.

En un segundo análisis se evaluaron los puntajes de los síntomas depresivos en función de las características sociodemográficas. La comparación de los grupos según su situación laboral y si tenían hijos se realizó mediante la prueba de la $t$ de Student, mientras que la comparación de los grupos según su estatus marital, la edad, el tipo de delito, los años de sentencia y la periodicidad de las visitas se realizó mediante análisis de varianza de una vía para grupos independientes. Cuando se obtuvieron valores significativos $(P \leq 0,05)$ en el análisis de varianza, se aplicó la prueba post hoc de StudentNewman-Keuls para identificar las diferencias significativas entre los grupos.

Las características sociodemográficas (estatus marital, número de hijos, situación laboral, frecuencia de visitas y tipo de delito) se presentan en frecuencias y porcentajes, mientras que las variables cuantitativas (edad, años de sentencia y puntajes de los síntomas depresivos) se presentan mediante los valores promedio y su error estándar.

Además de obtener la aprobación previa de las autoridades de los establecimientos penitenciarios y el consentimiento informado de las voluntarias participantes, esta investigación contó con la aprobación del Programa Estatal de la Mujer, entidad del Gobierno del Estado de Veracruz encargada de lograr un mejor nivel de vida para las mujeres vera- cruzanas mediante su desarrollo integral en los ámbitos educativo, cultural, económico, académico, social y laboral. Las mujeres con síntomas depresivos se refirieron a un programa de terapia ocupacional con la finalidad de reducir los síntomas y ofrecerles un seguimiento particular.

\section{RESULTADOS}

En el penal PV había 51 mujeres en prisión, de las que $32(62,7 \%)$ cumplieron con los criterios de inclusión y aceptaron participar voluntariamente en el estudio; mientras que de 21 mujeres del penal FSC, $19(90,5 \%)$ participaron en el estudio. No se encontraron diferencias significativas entre las mujeres de PV y FSC en cuanto a la edad $(P=0,22)$, el número de hijos $(P=0,84)$, el número de visitas $(P=0,91)$ y los años de sentencia $(P=0,94)$. Tampoco se encontraron diferencias significativas cuando las mujeres se agruparon según su estatus marital, situación laboral, tipo de delito y la frecuencia con que recibían visitas $(P>0,05)$. Todas las madres participantes en el estudio tenían a sus hijos fuera del penal (cuadro 1).
Todas las mujeres estudiadas en ambos penales presentaron síntomas depresivos. Las mujeres del penal PV tuvieron un puntaje significativamente menor de síntomas depresivos que las del penal FSC $(20,8 \pm 0,8$ frente a $25,2 \pm 0,9 ; P<0,001)$.

No hubo diferencias significativas entre las características sociodemográficas de las mujeres de los dos penales, por lo que el análisis siguiente se realizó con las mujeres de ambos penales en conjunto.

No se encontraron diferencias significativas en cuanto a la presencia de síntomas depresivos, por una parte, y la situación laboral de las mujeres dentro del penal $(P=0,18)$, su estatus marital $(\mathrm{F}[2,48]=0,47 ; P=0,62)$, la edad (F[3, $48]=2,93 ; P=0,06)$, el tipo de delito (F[5, $45]=0,86 ; P=0,51)$ y el tiempo de sentencia $(F[2,48]=0,42 ; P=0,65)$, por el otro. Sin embargo, el puntaje según la escala de Hamilton fue significativamente mayor en las mujeres con hijos que en las que no tenían hijos $(25,3 \pm 0,9$ frente a $21,8 \pm 1,3 ; P<0,05)$.

Se encontró un mayor puntaje de síntomas de depresión en las mujeres que no recibían visitas $(26,2 \pm 0,9)$, seguidas

CUADRO 1. Características sociodemográficas de las reclusas participantes en el estudio, México

\begin{tabular}{|c|c|c|}
\hline \multirow[b]{2}{*}{ Característica } & \multicolumn{2}{|c|}{ Establecimiento penitenciario $^{a}$} \\
\hline & $\begin{array}{l}\text { Pacho Viejo } \\
(n=32)\end{array}$ & $\begin{array}{l}\text { Fortaleza San Carlos } \\
\qquad(n=19)\end{array}$ \\
\hline Edad promedio, años \pm error estándar & $32,5 \pm 1,6$ & $36,2 \pm 2,8$ \\
\hline Sentencia promedio, años \pm error estándar & $14,8 \pm 2,1$ & $15,0 \pm 2,4$ \\
\hline \multicolumn{3}{|l|}{ Estatus marital (\%) } \\
\hline Con pareja en el penal & $18(56,3)$ & $9(47,4)$ \\
\hline Con pareja fuera del penal & $10(31,3)$ & $5(26,3$ \\
\hline Sin pareja & $4(12,4)$ & $5(26,3)$ \\
\hline \multicolumn{3}{|l|}{ Hijos (\%) } \\
\hline Con hijo fuera del penal & $22(68,7)$ & $18(94,7)$ \\
\hline Sin hijos & $10(31,3)$ & $1(5,3)$ \\
\hline Hijos por mujer, promedio \pm error estándar & $2,5 \pm 0,3$ & $3,2 \pm 0,5$ \\
\hline \multicolumn{3}{|l|}{ Situación laboral en el penal $(\%)^{\mathrm{b}}$} \\
\hline Empleadas & $26(81,3)$ & $16(84,3)$ \\
\hline No empleadas & $6(18,7)$ & $3(15,7)$ \\
\hline \multicolumn{3}{|l|}{ Visitas (\%) } \\
\hline Semanal & $8(25,0)$ & $1(5,3)$ \\
\hline Quincenal & $6(18,7)$ & $1(5,3)$ \\
\hline Mensual & $4(12,6)$ & $4(21,1)$ \\
\hline$<1$ al mes & $8(25,0)$ & $6(31,5)$ \\
\hline No recibe visitas & $6(18,7)$ & $7(36,8)$ \\
\hline Visitantes por mujer, promedio \pm error estándar ${ }^{c}$ & $3,6 \pm 0,4$ & $3,5 \pm 1,2$ \\
\hline \multicolumn{3}{|l|}{ Delito sancionado (\%) } \\
\hline Tráfico de drogas & $8(25,0)$ & $5(26,4)$ \\
\hline Homicidio & $6(18,7)$ & $9(47,3)$ \\
\hline Secuestro & $3(9,4)$ & $2(10,5)$ \\
\hline Robo & $2(6,3)$ & $0(0,0)$ \\
\hline Fraude & $5(15,6)$ & $1(5,3)$ \\
\hline Otros $^{d}$ & $8(25,0)$ & $2(10,5)$ \\
\hline
\end{tabular}

a No se encontraron diferencias significativas entre las características sociodemográficas de las mujeres de ambos penales. ${ }^{b}$ Abarca actividades como coser, bordar, lavar, planchar, vender comida y hacer manualidades y artículos de talabartería.

c Se excluyeron las mujeres que no recibían visitas.

d Entre otros, portación ilegal de armas, encubrimiento, lesiones y violación equiparada. 
FIGURA 1. Puntajes de depresión de los grupos de reclusas según la periodicidad de las visitas recibidas, México

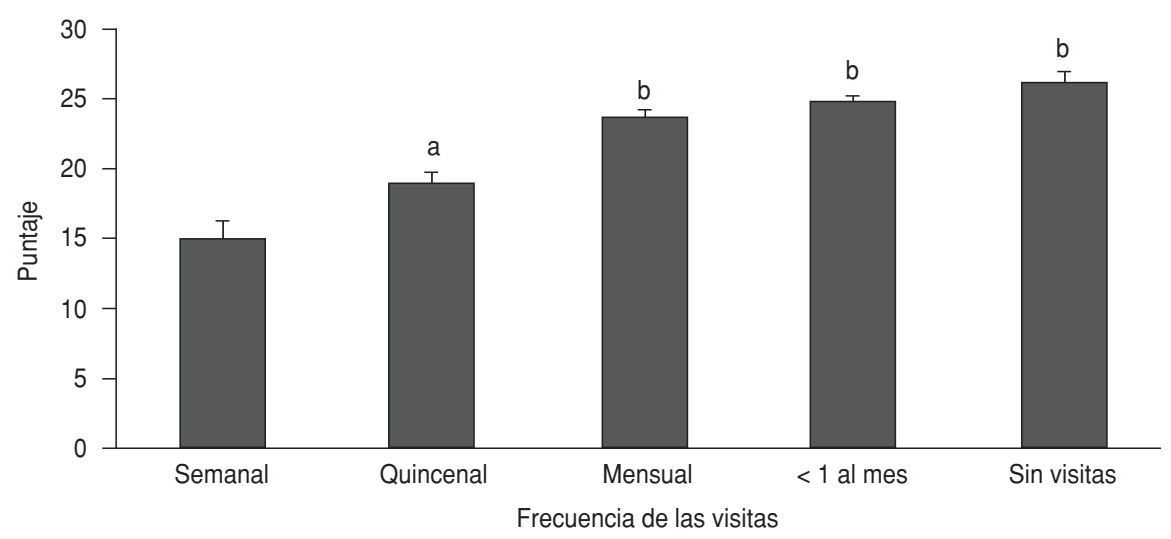

a Diferencia significativa con respecto a los grupos de mujeres que recibían visitas semanales, mensuales, $<1$ al mes y sin visitas. Prueba post hoc de Student Newman-Keuls, $P<0,0001$.

${ }^{b}$ Diferencia significativa con respecto a los grupos de mujeres que recibían visitas semanales y quincenales. Prueba post hoc de Student Newman-Keuls, $P<0,005$.

de las que recibían menos de una visita al mes $(24,8 \pm 0,5)$, las que recibían visitas mensuales $(23,6 \pm 0,6)$ y las que recibían visitas quincenalmente $(19,0 \pm 0,7)$; el menor puntaje lo presentó el grupo de mujeres que recibían visitas semanalmente $(15,0 \pm 1,3)$, con diferencia significativa entre este grupo y los restantes $(\mathrm{F}[4,46]=30,7 ; P<0,0001)$ (figura 1 ).

La prueba post hoc reveló que no existían diferencias significativas entre los grupos de mujeres que no recibían visitas y las que recibían visitas mensuales o menos de una visita al mes; sin embargo, estos tres grupos presentaron mayor puntaje de síntomas depresivos que los grupos de mujeres que recibían visitas semanal o quincenalmente $(P<0,05)$.

\section{DISCUSIÓN}

El trastorno depresivo se presenta con un marcado patrón sexual en la población general y su prevalencia es casi dos veces más frecuente en las mujeres (10$25 \%)$ que en los hombres (5-12\%) (27-30). Según la Encuesta Nacional de Epidemiología Psiquiátrica (31), la prevalencia de depresión mayor en la población general de México es de 3,3\% (mujeres: 4,5\%; hombres: $2,0 \%)$ y la de depresión menor es de 1,5\% (mujeres: 2,3\%; hombres: $0,6 \%$ ). Otros estudios señalan una prevalencia de depresión en la población mexicana adulta de $5,8 \%$ en mujeres y $2,5 \%$ en hombres (32).

Con respecto a la población encarcelada, en una revisión sistemática de 62 estudios -que abarcaba un total de 22790 prisioneros en 12 países - se encontró una prevalencia de trastorno depresivo mayor más elevada (12\% en mujeres y $10 \%$ en hombres) que en la población general (6), aunque otros autores han encontrado prevalencias mayores de hasta $20 \%$ en mujeres y $14 \%$ en hombres (8). Esto concuerda con investigaciones en que se ha encontrado una mayor frecuencia de depresión en las mujeres que en los hombres encarcelados $(33,34)$. En México, los pocos estudios realizados en la población penal han mostrado una elevada frecuencia de trastornos depresivos en mujeres (entre $57,1 \%$ y $72,7 \%)(16,17,35)$. En el presente estudio, se encontró también una alta frecuencia de los síntomas depresivos en todas las participantes, lo que concuerda con la alta frecuencia de síntomas depresivos observada en prisiones de otros países $(6,20)$ y de México $(17,35)$.

El encarcelamiento, tanto de hombres como de mujeres, es una situación altamente estresante que puede contribuir a la alta frecuencia de trastornos mentales encontrada en la población recluida en penales, entre ellos la ansiedad y la depresión $(20,36,37)$. Se ha encontrado que tener hijos puede ser un factor adicional que condiciona la alta frecuencia de síntomas depresivos en las mujeres que guardan prisión. En un estudio reciente realizado en dos prisiones para mujeres de la Ciudad de México se encontró que la frecuencia general de episodios depresivos fue de $62 \%$, superior en las mujeres que tenían hijos y más elevada aún en las que tenían hijos menores de 18 años (17). En el presente estudio, las reclusas que eran madres tenían sus hijos fuera del penal, lo que podría contribuir, entre otros factores, a la alta frecuencia de los síntomas depresivos encontrada en las madres de ambos penales.

Según datos obtenidos en otros países, las madres que guardan prisión son más vulnerables de padecer algún trastorno mental que las reclusas sin hijos $(10,18)$, al parecer por el dolor moral que experimentan las madres por ver limitado el contacto con sus hijos $(10,16,18)$. Además, las madres en prisión experimentan un mayor estrés por sentir reducidos su identidad como madres y el control sobre sus hijos, así como por el sufrimiento que les genera pensar en la información que recibirán sus hijos sobre ellas y su conducta criminal (38). Estos factores tienen un gran impacto, no solo sobre el estado emocional de las madres encarceladas, sino también sobre su autoestima, autodefinición e identidad como mujer (39). En 1992, Fogel y Martin (40) encontraron que en la primera semana de encarcelamiento, las mujeres con hijos o sin ellos tienen niveles de ansiedad elevados que, en muchos casos, preceden a la depresión; seis meses después, sin embargo, la ansiedad disminuye solamente en las mujeres sin hijos y se mantiene alta en las que son madres. Estos autores sugieren que este efecto se debe a la separación forzada de sus hijos, así como a la pérdida del contacto con familiares y 
personas de su entorno social previo al encarcelamiento $(40,41)$.

Además, un bajo nivel de apoyo social hacia las mujeres en prisión, representado por un insuficiente contacto con sus familiares y amigos, repercute negativamente sobre su bienestar físico y mental (42-45). En el presente estudio, las mujeres que recibían visitas semanalmente tuvieron los menores puntajes de síntomas depresivos, mientras que las que no recibían visitas o las recibían con menor frecuencia presentaron los mayores puntajes. Se ha informado que el apoyo que reciben las mujeres en prisión por parte del esposo, los familiares o los amigos es clave para su bienestar físico y mental. Las personas encarceladas que mantienen contacto con sus familiares y amigos por teléfono, cartas o visitas se ajustan más adecuadamente al contexto carcelario y presentan menos trastornos de salud física y mental $(16,46)$.

En un estudio reciente se informó que las mujeres encarceladas que recibieron visitas en el mes previo a la investigación valoraron positivamente su salud física y mental, mientras que las que no recibieron visitas la valoraron negativamente $y$ tuvieron una tendencia a presentar más episodios de depresión que sus pares que habían recibido visitas (16). Esto es consistente con otros estudios que señalan que el inadecuado apoyo social en personas aisladas, en ambientes confinados o con enfermedades incapacitantes contribuye a su deterioro emocional y físico, mientras que el apoyo social y familiar contribuye a su mejoramiento físico, emocional y mental. Además, el apoyo social mantiene bajos los índices de ansiedad y depresión, aún en condiciones de estrés psicosocial (47-55). Esto podría explicar los altos puntajes de síntomas depresivos encontrados en las participantes en el presente estudio que no re- cibían visitas o en las que recibían menos de una visita al mes, en comparación con las mujeres que recibían visitas semanalmente, cuyos puntajes de depresión fueron los menores. El contacto social podría contribuir a un ajuste más adecuado al contexto carcelario y a reducir los efectos negativos del estrés psicosocial sobre la salud física y mental de las mujeres que guardan prisión.

Por otro lado, algunos autores señalan que el inadecuado diseño arquitectónico de las cárceles, entre otros aspectos, puede influir en el deterioro de la salud física y mental de las personas que se encuentran en prisión (56-57). En este sentido, las condiciones arquitectónicas del penal FSC podrían contribuir al mayor puntaje de síntomas depresivos de sus reclusas con respecto a las del penal PV, ya que el primero no se diseñó como centro de readaptación social y carece de los espacios necesarios para este fin, una posibilidad que debe ser abordada en estudios específicos.

El presente estudio tiene algunas limitaciones. Primero, es importante señalar que en esta investigación solamente participó $62,7 \%$ de las mujeres del penal PV, mientras que del penal FSC participó 90,5\%; se desconoce la situación del $47,3 \%$ restante de las mujeres del penal PV. Segundo, la escala de depresión de Hamilton solo permite detectar los síntomas depresivos que sufren las mujeres estudiadas, sin identificar el trastorno depresivo en sí. Tercero, en este estudio se encontró que las madres encarceladas tuvieron mayor puntaje de síntomas depresivos que las participantes que no tenían hijos, lo que podría deberse al estrés generado por la situación del cuidado parental. Sin embargo, no se aplicó un instrumento específico para evaluar este tipo de estrés. Cuarto, en lo relativo a la interacción de las mujeres en prisión con sus familiares y amigos, solo se analizó la frecuencia de las visitas recibidas, pero no se aplicó un instrumento que evaluara la disponibilidad del apoyo social bajo distintas circunstancias o el grado de satisfacción de estas mujeres con el apoyo recibido. Del mismo modo, no se consideró el nivel socioeconómico de las mujeres estudiadas y sus familiares, lo que podría influir en algunas de las variables asociadas, como la periodicidad de las visitas.

A pesar de estas limitaciones, se puede afirmar que se encontró una elevada frecuencia de síntomas depresivos en la muestra estudiada de mujeres recluidas en las dos prisiones estudiadas. El tener hijos y el recibir visitas con poca frecuencia pueden influir en la aparición y la intensidad de los síntomas depresivos en las mujeres encarceladas. Para contribuir con el bienestar físico y mental de las mujeres que se encuentran en prisión, los programas de readaptación social deben fomentar el contacto permanente con sus hijos, familiares y amigos. Se deben diseñar estudios específicos para evaluar el impacto de estas medidas sobre la frecuencia y la intensidad de los síntomas depresivos.

Agradecimientos. Los autores agradecen a las voluntarias que participaron en esta investigación, así como al Programa Estatal de la Mujer y a la Dirección General de Prevención y Readaptación Social, ambos del estado de Veracruz, México, por el apoyo administrativo brindado para la realización del presente estudio. Para esta investigación, el primer autor recibió una beca del Instituto Mexicano de la Juventud y el segundo autor recibió apoyo parcial del Sistema Nacional de Investigadores (No. 32753) y del Programa de Mejoramiento del Profesorado (No. 103,5/05/1955, Universidad Veracruzana, PTC-155).

\section{REFERENCIAS}

1. Walmley R. World prison population list. 2. ${ }^{\mathrm{a}}$ ed. London: Home Office Research, Development and Statistics Directorate; 2000.

2. Greenfeld L, Snell TL. Bureau of Justice Statistics special report: women offenders. Washington, D.C.: Department of Justice Office of Justice Program; 1990. (BJS Document 175688.)

3. Edwards TA. Female offenders: special needs and southern state challenges. Atlanta: South- ern Legislative Conference of the Council of State Governments; 2000.

4. Bloom B, Owen B, Covington S. Genderresponsive strategies: research, practice, and guiding principles for women offenders. Washington, D.C.: National Institute of Corrections; 2003.

5. Gunter TD. Incarcerated women and depression: a primer for the primary care provider. J Am Med Womens Assoc. 2004;50:107-12.
6. Fazel S, Danesh J. Serious mental disorder in 23000 prisoners: a systematic review of 62 surveys. Lancet. 2002;359:545-50.

7. Van der Wurff FB, Beekman ATF, Dijkshoorn $\mathrm{H}$, Spijker JA, Smits CHM, Stek ML, et al. Prevalence and risk-factors for depression in elderly Turkish and Moroccan migrants in the Netherlands. J Affect Disord. 2004;83:33-41.

8. Butler T, Allnutt S, Cain D, Owens D, Muller C. Mental disorder in the New South Wales 
prisoner population. Aust N Z J Psychiatr. 2005;39:407-13.

9. Lewis C. Treating incarcerated women: gender matters. Psychiatr Clin North Am. 2006; 29:773-89.

10. Kurshan N. Women and imprisonment in the U.S.: history and current reality. Austin, TC: Monkeywrench Press; 1996. Hallado en http:// www.prisonactivist.org/archive/women/ women-and-imprisonment.html. Acceso el 10 de agosto de 2009.

11. Lagarde M. Los cautiverios de las mujeres: madre, esposa, monjas, putas, presas y locas. México, D. F.: Universidad Nacional Autónoma de México; 1993.

12. Azaola E. Las mujeres olvidadas. México, D.F.: Colegio de México, Comisión Nacional de Derechos Humanos; 1996.

13. Champion D. Corrections in the United States: a contemporary perspective. 3 . $^{\mathrm{a}}$ ed. Upper Saddle River, NJ: Prentice-Hall; 2001.

14. Houck KDF, Loper AB. The relationship of parenting stress to adjustment among mothers in prison. Am J Orthopsychiatry. 2002;72: 548-58.

15. Cassano P, Fava M. Depression and public health. J Psychosom Res. 2002;53:849-57.

16. Galván J, Romero M, Rodríguez EM, Durand A, Colmenares E, Saldívar G. La importancia del apoyo social para el bienestar físico y mental de las mujeres reclusas. Salud Mental. 2006;29:68-74.

17. Colmenares-Bermúdez E, Romero-Mendoza MP, Rodríguez-Ruíz EM, Durand-Smith AL, Saldívar-Hernández GJ. Female depression and substance dependence in the México City penitentiary system. Salud Mental. 2007;30:53-61.

18. Poehlmann J. Incarcerated mothers' contact with children, perceived family relationships, and depressive symptoms. J Fam Psychol. 2005; 19:350-7.

19. Kessler RC, Price RH, Wortman CB. Social factors in psychopathology: stress, social support, and coping processes. Ann Rev Psychol. 1985;36:531-72.

20. Tye CS, Mullen PE. Mental disorders in female prisoners. Aust N Z J Psychiatr. 2006;40: 266-71.

21. Ramos-Brieva JA, Cordero A. Validación de la versión castellana de la escala de Hamilton para la depresión. Actas Luso-Esp Neurol Psiquiat. 1986;14:324-44.

22. Hamilton M. Development of rating scales for primary depressive illness. Br J Soc Clin Psychol. 1967;6:278-96.

23. Bagby RM, Ryder AG, Schuller DR, Marshall MB. The Hamilton depression rating scale: has the gold standard become a lead weight? Am J Psychiatr. 2004;161:2163-77.

24. Nicolini H, Fresán A. Escalas de evaluación de los trastornos afectivos. En: Apiquián R, Fresán A, Nicolini H, eds. Evaluación de la sicopatología. Escalas en español. México, D.F.: JGH Editores; 2000. Pp. 49-59.

25. Ruiz-Flores LG, Colín-Piana RF, CorlayNoriega ISY, Lara-Muñoz MC, Dueñas-Tentori HJ. Trastorno depresivo mayor en México: la relación entre la intensidad de la depresión, los síntomas físicos dolorosos y la calidad de vida. Salud Mental. 2007;30:25-32.

26. Hurley W, Dunne MP. Psychological distress and psychiatric morbidity in women prisoners. Aust N Z J Psychiatr. 1991;25:461-70.

27. Breslau N, Schultz L, Peterson E. Sex differences in depression: a role for preexisting anxiety. Psychiatr Res. 1995;58:1-12.

28. Piccinelli M, Wilkinson G. Gender differences in depression: critical review. Br J Psychiatr. 2000;177:486-92.

29. Williams JBW, Spitzer RL, Linzer M, Kroenke $\mathrm{K}$, Hahn SR, deGruy FV, et al. Gender differences in depression in primary care. Am J Obstet Gynecol. 1995;173:654-9.

30. Kessler RC. Epidemiology of women and depression. J Affect Disord. 2003;74:5-13.

31. Medina-Mora ME, Borges G, Lara-Muñoz C, Benjet C, Blanco-Jaime J, Fleiz-Bautista C, et al. Prevalencia de trastornos mentales y uso de servicios: resultados de la encuesta nacional de epidemiología psiquiátrica en México. Salud Mental. 2003;26:1-16.

32. Bello M, Puentes-Rosas E, Medina-Mora ME, Lozano R. Prevalencia y diagnóstico de depresión en población adulta en México. Salud Publica Mex. 2005;47:4-11.

33. Leibenluft E. Gender differences in major depressive disorder and bipolar disorder. CNS Spectr. 1999;4:25-33.

34. Zlotnick C, Clarke JG, Friedmann PD, Roberts MB, Sacks S, Melnick G. Gender differences in comorbid disorders among offenders in prison substance abuse treatment programs. Behav Sci Law. 2008;26:403-12.

35. Hernández-Zamora ZE, Márquez-Barradas ML. Evaluación de la salud mental de la población de internas de un centro de readaptación social. Psicol Salud. 1999;14:101-10.

36. Jordan BK, Schlenger WE, Fairbank JA, Caddell JM. Prevalence of psychiatric disorders among incarcerated women. II. Convicted felons entering prison. Arch Gen Psychiatr. 1996;53:513-9.

37. Bonner RL. Stressful segregation housing and psychosocial vulnerability in prison suicide ideators. Suicide Life Treat Behav. 2006;36: 250-4.

38. Fox JG. Women in prison: a case study in the social reality of stress. In: Johnson $\mathrm{R}$, Toch $\mathrm{H}$, eds. The pain of imprisonment. Beverly Hills, CA: Sage; 1982. Pp. 205-20.

39. Baunach PJ. Mothers in prison. New Brunswick, NJ: Transaction Books; 1985.

40. Fogel CI, Martin SL. The mental health of incarcerated women. West J Nurs Res. 1992;14: 30-47.

41. Fogel CI. Hard time: the stressful nature of incarceration for women. Issues Ment Health Nurs. 1993;14:367-77.

42. Bayse DJ, Allgood SM, Wyk PH. Family life education: an effective tool for prisoner rehabilitation. Fam Rel. 1991;40:254-7.

43. Carlson BE, Cervera JJ. Incarceration, coping, and support. Soc Work. 1991;36:279-85.

44. Muraskin R. The pains of imprisonment. Long-term incarceration effects on women in prison. In: It's a crime: women and justice.
Upper Saddle River, NJ: Prentice Hall Contemporary Justice; 2000. (Series 205-213.)

45. Spotts EL, Neiderhiser JM, Ganiban J, Reiss D, Lichtenstein P, Hansson K, et al. Accounting for depressive symptoms in women: a twin study of associations with interpersonal relationships. J Affect Disord. 2004;82:101-11.

46. Bennett LA. What has happened to prison visiting? Current use of rehabilitative tool. In: Gottfredson DM, Goldkamp JS, eds. Proceedings of the 39th Meeting of the American Society of Criminology. Montreal: American Society of Criminology; 1987. Pp. 48.

47. Hough ES, Brumitt GA, Templin TN. Social support, demands of illness, and depression in chronically illness urban women. Health Care Women Int. 1999;20:349-62.

48. Martínez-Schallmoser L, Telleen S, MacMullen NJ. The effect of social support and acculturation on postpartum depression in Mexican American women. J Transcult Nurs. 2003;14:329-38.

49. Palinkas LA, Jonson JC, Boster JS. Social support and depressed mood in isolated and confined environments. Acta Astronautica. 2004; 54:639-47.

50. Chi I, Yip PS, Chiu HF, Chou KL, Chan KS, Kwan CW, et al. Prevalence of depression and its correlates in Hong Kong's Chinese older adults. Am J Geriatr Psychiatr. 2005;13:409-16.

51. Compton MT, Thompson NJ, Kaslow NJ. Social environment factors associated with suicide attempt among low-income African Americans: the protective role of family relationships and social support. Soc Psychiat Epidemiol. 2005;40:175-85.

52. Yen $\mathrm{WJ}$, Lundeen $\mathrm{S}$. The association between meaning of caregiving, perceived social support and level of depression of Taiwanese caregivers of mental ill patients. Int J Psychiatr Nurs Res. 2006;12:1378-92.

53. Zyrianova Y, Kelly BD, Gallagher C, McCarthy C, Mohillo MG, Sheehan J, et al. Depression and anxiety in rheumatoid arthritis: the role of perceived social support. Ir J Med Sci. 2006;175:32-6.

54. Plaisier I, de Bruijn JG, de Graaf R, Have MT, Beekman AT, Penninx BW. The contribution of working conditions and social support to the onset of depressive and anxiety disorders among male and female employees. Soc Sci Med. 2007;64:401-10.

55. González Ramírez MT, Landero Hernández R, Ruiz Díaz MA. Modelo estructural predictor de la salud mental y física en mujeres. Rev Panam Salud Publica. 2008;23(2):101-8.

56. Kaufman E. The violation of psychiatric standards of care in prisons. Am J Psychiatr. 1980; 137:566-70.

57. De Viggiani N. Unhealthy prisons: exploring structural determinants of prison health. Sociol Health Illn. 2007;29:115-35.

Manuscrito recibido el 5 de junio de 2008. Aceptado para publicación, tras revisión, el 18 de noviembre de 2008. 
ABSTRACT Objectives. To determine the prevalence of depressive symptoms among a sample of women held in two Mexican prisons and to identify the sociodemographic factors associated with these symptoms.

Sociodemographic factors associated with symptoms of depression in a sample of women held at two prisons in Mexico
Methods. An exploratory, descriptive study was conducted of women 18-50 years of age in two prisons in the state of Veracruz, Mexico: the Pacho Viejo (PV) facility and Fortaleza San Carlos (FSC) facility. The Hamilton scale for depressive illness was applied and relationships were found between the participants' scores and their sociodemographic traits (age, marital status, number of children, jail work assignment, and number/frequency of visitors), legal status, type of crime committed, and length of sentence. The analysis employed Student's T test, Chi-squared test, and StudentNewman-Keuls' post hoc test, depending on the variable.

Results. All of the women studied at both facilities presented with symptoms of depression. Women at the PV facility had a lower score for depressive symptoms than those at FSC $(20.8 \pm 0.8$ versus $25.2 \pm 0.9 ; P<0.001)$. A higher score was found among women with children than among those without children $(25.3 \pm 0.9$ versus $21.8 \pm 1.3$; $P<0.05)$; the group of women receiving weekly visits scored $(15.0 \pm 1.3)$ significantly lower than the other groups $(\mathrm{F}[4.46]=30.7 ; P<0.0001)$. No significant differences were found to be related to marital status, work assignment, age, type of crime, or the length of sentence.

Conclusions. Symptoms of depression were prevalent among the study sample, and were most powerfully related to having children and infrequent visits. Social adaptation programs should encourage continuous contact between women and their children, family, and friends.

Key words Women's health; prisons; depressive disorder; social support; Mexico. 